\title{
Estrategias de distribución para la comercialización de Pisco en el mercado interno: Asociación de productores de Ica
}

\author{
Distribution strategies for marketing Pisco in the domestic market: Association of producers of Ica
}

\author{
Karina Marlen Yachi Del Pino ${ }^{1}$
}

\begin{abstract}
Resumen
El presente estudio se ha desarrollado con el objetivo de describir el panorama que enfrenta el sector pisquero, específicamente el de los pequeños productores de la zona de Ica, en ese sentido se aborda un diagnóstico que permite ver las principales características que enfrentan, así como las condiciones del producto en el mercado al cual se dirige. Actualmente las dificultades en la comercialización, los ha colocado en un lento crecimiento, es así que enfrentan problemas en la colocación de sus productos, desconocen el mercado y no desarrollan estrategias de comercialización. Por ello, se pretende abrir el conocimiento de la realidad que enfrentan, al identificar un conjunto de acciones válidas que servirán para establer acciones que permitirán mejorar las condiciones de comercialización en el mercado interno.
\end{abstract}

Palabras clave: Comercialización; distribución; estrategias; pisco; mercado interno.

\begin{abstract}
The present investigation has been developed with the objective of describing the panorama that confronts the sector pisquero, specifically the one of the small producers of the zone of Ica, in that sense a diagnosis is approached that allows to see the main characteristics that face, as well as the conditions of the product in the market to which it is directed. Currently the difficulties in marketing, has placed them in a slow growth, so they face problems in the placement of their products, they ignore the market and do not develop marketing strategies. Therefore, it is intended to open up the knowledge of the reality that they face, by identifying a set of valid actions that will serve to establish actions that will improve the conditions of commercialization in the domestic market.
\end{abstract}

Keywords: Marketing; distribution; strategies; pisco; internal market.

\section{Introducción}

La presente investigación justifica su desarrollo en la pretensión de aportar en la comprensión y el conocimiento del funcionamiento de la comercialización del sector pisquero del país, específicamente en cuanto a las estrategias de distribución existente, sobre cuyos caracteres se ve reflejado su alcance y resultados en el mercado que atiende. Es preciso destacar que el estudio realizado es conveniente para establecer un eje de orientación para las empresas medianas y pequeñas que actualmente operan bajo un enfoque tradicional y cuyo ritmo de crecimiento es moderado - lento.

La importancia del estudio se centra en los hallazgos, los cuales permitirán una mejor toma de decisiones, tanto al conjunto de productores promotores de la marca colectiva como a los medianos y pequeños empresarios del pisco, cuyo principal reto sigue siendo la comercialización de sus productos, el uso, desarrollo y consolidación de sus marcas.

La problemática existente en la actividad pisquera es compleja debido a la presencia de una heterogénea composición de empresas, por tamaño, años de permanencia en el mercado, desarrollo del producto, posicionamiento y/o capacidad de ingreso a nuevos mercados, vienen desarrollando una actividad empresarial desarticulada. Sin embargo, a través de la asociatividad han resuelto problemas de accesibilidad. De este modo las diversas asociaciones de productores pisqueros con denominación de origen han logrado llegar al 2014 a trabajar cinco marcas colectivas (Gran Medalla, Orovilca. Willca, Doce Sangres, y El Arco de mi Frontera).

Las cuales no logran colocar su oferta de forma ágil y con metas cada vez más ambiciosas en un ambiente competitivo, existe la necesidad de alcanzar niveles de efectividad en las relaciones: Empresa - Intermediarios - Consumidor Final, utilizando distintos flujos de intercambio y múltiples formas de llegar al consumidor y/o cliente. Un tema pendiente es el ingreso al mercado interno de forma sostenida (Díaz, 2006).

El objetivo de la presente investigación fue identificar las principales características de la realidad del sector y las oportunidades potenciales para los pequeños productores de la zona de Ica, describir la composición de los canales de comercialización para el pisco y finalmente se prende establecer los lineamientos para una adecuada gestión comercial en Lima Metropolitana que permitirá superar las dificultades de ingreso y/o generar su dinamismo. 


\section{Materiales y métodos}

El presente estudio es una investigación no experimental aplicada, debido a que no se realizaron pruebas de laboratorio sino el análisis del comportamiento desde la experiencia de la Asociación de Productores de Ica. Es descriptiva, porque describe los principales caracteres de la comercialización y relaciones en la distribución en el mercado interno con ayuda de herramientas confiables que permitan la valoración de resultados (Hernández, 1997). Se consideran dos niveles: Investigación Exploratoria, delimita el problema con base en la búsqueda de elementos elevantes al tema que permitan un panorama amplio del contexto. E investigación Concluyente, que permitió los hallazgos que han llevado al desarrollo de la presente redacción y las afirmaciones que se realizan. Se desarrolla la aplicación de las herramientas (encuesta, entrevista y observación). Se usaron Cuestionarios para la realización de encuestas, documento elaborado a partir de interrogantes surgidas desde la investigación. Entrevista, elaborada a partir de una guía de indagación dirigido a los productores de líderes del sector. Y observación, que sirvió para el análisis de los puntos de venta de diferentes canales.

\section{Resultados y discusión}

Los patrones de comercio se centran en tres clasificaciones: consumo local, consumo regional y exportación (está a través de un acopiador y la exportación directa a través de la asociatividad). El Pisco ha mostrado crecimiento en los mercados donde participa (Figura 1), así como en el número de empresas que conforman el sector, su desarrollo ha sido interesante destacándose así que la principal empresa productora - exportadora al 2014 fue "Destilería La Caravedo S.R.L" con su marca "Porton"; que es la única que viene desarrollando una campaña promocional agresiva en los mercados internacionales. Lama (2006), manifiesta que la vid para producción de pisco ocupa el $12 \%$ de los cultivos, una cifra que se espera crezca a partir del impulso de organismos como el Centro de Innovación Tecnológica de la Vid - CITEvid. En el sector productivo la fragmentación de la propiedad ocasionó que existan propietarios de menos de una hectárea.

Se puede afirmar con claridad que el mercado del pisco ha logrado un desarrollo parcial, producto del crecimiento en el mercado de exportación que convive con un lenta penetración de los pequeños productores artesanales cuyo único mercado es el interno, el mismo que un $90 \%$ es local, es decir se centra en la comercialización que no logra un crecimiento a nivel nacional, en este sentido Achura (2005) recalcó que es importante reconocer que la demanda y el tiempo de entrega no se pueden conocer con seguridad, por lo tanto se debe plantear una situación en la que haya suficiente existencia disponible para satisfacer la demanda, la misma que debiera ser estimulada para lograr un mayor consumo per cápita.

Actualmente en el mercado los retails o centros de distribución al menudeo han permitido que numerosas empresas alcancen sus metas comerciales (Monge, 2003).
Las tendencias de la comercialización se manifiestan por la concentración de los procesos de distribución en grandes empresas de cobertura nacional, denominadas retails, en el uso de tecnologías modernas física y electrónica, la confianza en el proveedor se convierte un factor clave en la relación comercial, necesidades de bajos costos en los canales de distribución, exigencias con respecto al medio ambiente y uso inteligente de agroquímicos, exigencias sobre seguimiento y trazabilidad (rastreo) de las operaciones y de los productos, concentración de los canales de comercialización de Hiper y mega mercados, en este escenario los distribuidores y consumidores comienzan a acostumbrarse al abastecimiento durante todo el año (year-round) las 24 horas del día. En los países en desarrollo se tiende a la exportación de productos de la agroindustria.

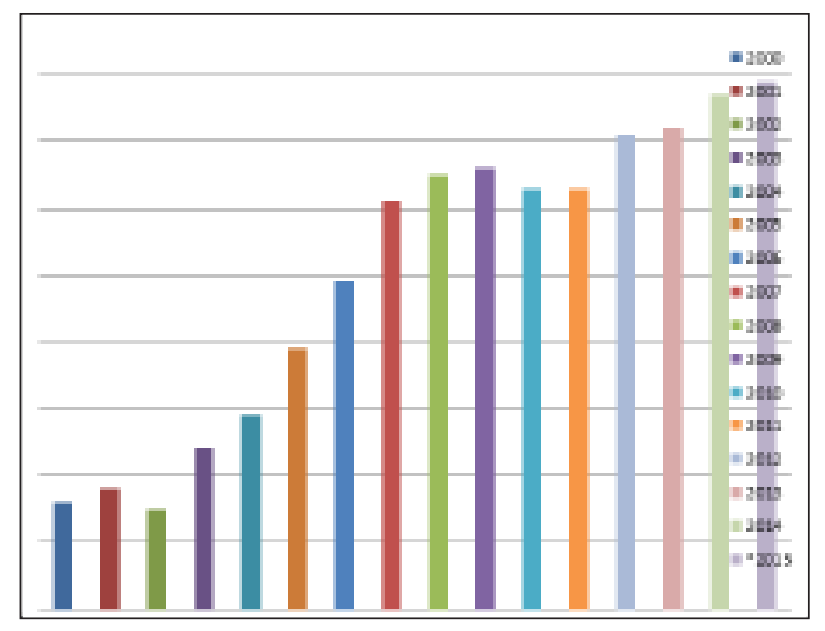

Figura 1. Volúmenes de Producción de Pisco. Período 2000-2015

Fuente: CITE Agroindustrial, SIN, Produce, Sunat.

Sin embargo, hace falta una dosis eficiente de calidad en los diferentes procesos productivos y de comercialización, son las recomendaciones que realiza Calderón (2000) en su investigación en donde señala que las pequeñas y medianas empresas pueden utilizar tecnologías muy sencillas para dar valor agregado al producto, considerando además que los canales de comercialización son muy importantes para que los productos lleguen a los consumidores, en este sentido es necesario seleccionar adecuadamente a nuestros "partners" o socios comerciales. Para los productores de Pisco de la zona de Ica la actividad de comercialización que realizan de forma individual les genera diferentes tipos de limitaciones como el acceso a mercados por volúmenes, ausencia de economías de escala, sobre costos en el trasporte y ausencia de un plan de comunicación por financiamiento entre otros; es así que se visualiza la comercialización conjunta por medio de cooperativas y otras formas asociativas creadas por los productores es una de las posibles estrategias para la apropiación de los excedentes por parte de estos y la eliminación de las barreras por un lado pero además la mejora sustancial de los ingresos ya que se van realizando actividades que los intermediarios las hacían por un margen de distribución. 


\section{Principales rasgos del sector}

La estructura de producción es diversificada, en uvas, tipo de pisco y vino. A partir del año 2002 coincidiendo con la instalación del centro de Innovación Tecnológica Vitivinícola (Cite-Vid) en Ica es que presenta un fuerte dinamismo en el sector productivo, que se refleja en el posicionamiento del pisco reconocido como la bebida bandera del país. El Citevid presta servicios y transferencia tecnológica para las pymes del espacio geográfico con D.O. (Tabla 1) hoy convertido en el Cite Agroindustrial.

Tabla 1. Las empresas con D.O. en la zona de Ica

\begin{tabular}{|c|c|c|c|c|}
\hline $\begin{array}{c}\text { Zona } \\
\text { geográfica }\end{array}$ & $\begin{array}{l}\text { Producto } \\
\text { principal }\end{array}$ & Producción & $\begin{array}{l}\text { Mercado } \\
\text { principal }\end{array}$ & $\begin{array}{l}\text { Marcas } \\
\text { con D.O }\end{array}$ \\
\hline $\begin{array}{c}\text { Departamento } \\
\text { de Ica }\end{array}$ & $\begin{array}{l}\text { Pisco } \\
\text { Vino } \\
\text { Uvas }\end{array}$ & 7762 Has & $\begin{array}{c}\text { Local regional } \\
\text { Nacional } \\
\text { Internacional }\end{array}$ & 501 \\
\hline
\end{tabular}

Fuente: Minag (2008) y Cite Agroindustrial (2014).

La concentración es un rasgo empresarial en el caso del pisco convive paralelamente con su alto nivel de fragmentación productiva que la hace vulnerable. Las empresas pisqueras de mayor crecimiento se concentran en un grupo reducido de marcas líderes, típica características de este sector, el mismo que según expertos en el tema (Cite Agroindustrial, 2014) paso en el año 2000 de representar 16 marcas a superar en el año 2014 nada menos que a 470 marcas registradas y 501 con denominación de origen.

El mercado interno actualmente se ha vuelto atractivo sin dejar de lado el internacional. Díaz (2006) señala que las organizaciones deben ser competitivas internamente para poder serlo externamente. Construir la competitividad interna es el gran reto de la gerencia moderna del pisco.

La asociación de productores de pisco de la zona de IcaApropica - cuenta con más de 100 socios de las localidades de La Tinguiña, Ica, Los Aquijes, Los Molinos, Ocucaje, Pachacutec, Parcona, Pueblo Nuevo, Salas, San Juan Bautista, Santiago, Subtanjalla, Tate, Yauca del Rosario, siendo el distrito con mayor volumen de producción de Pisco: Santiago, Salas de Guadalupe e Ica cuyo principal mercado es local.

El crecimiento de las marcas presentes en el mercado se ha dado a través del canal de retail en el que actualmente se exhiben más de 50 marcas, destacándose un grupo de 12 líderes las cuales tiene diferentes presentaciones en tamaño y variedad del pisco: Tacama, Ocucaje, Quierolo, Biondi, Porton, entre otras.

Las asociaciones como Apropica más allá de congregar a los más prestigiosos pequeños productores su labor es meritoria en términos de calidad y un acercamiento a los estándares y normas. Sin embargo, en ella se reconoce desde ya hace una década un débil ingreso a los mercados.

Según investigaciones realizadas sobre sobre el consumidor limeño, este consume pisco en un 46\% mientras que encontramos resistencia a su consumo en un $54 \%$ de la población, siendo una de las causas la percepción del elevado nivel de alcohol, que sumado a un desconocimiento en la elaboración de cócteles generan una respuesta negativa, sin embargo a nivel de los segmentos A, B y C, su consumo en promedio es del 58\% (Figura 2).

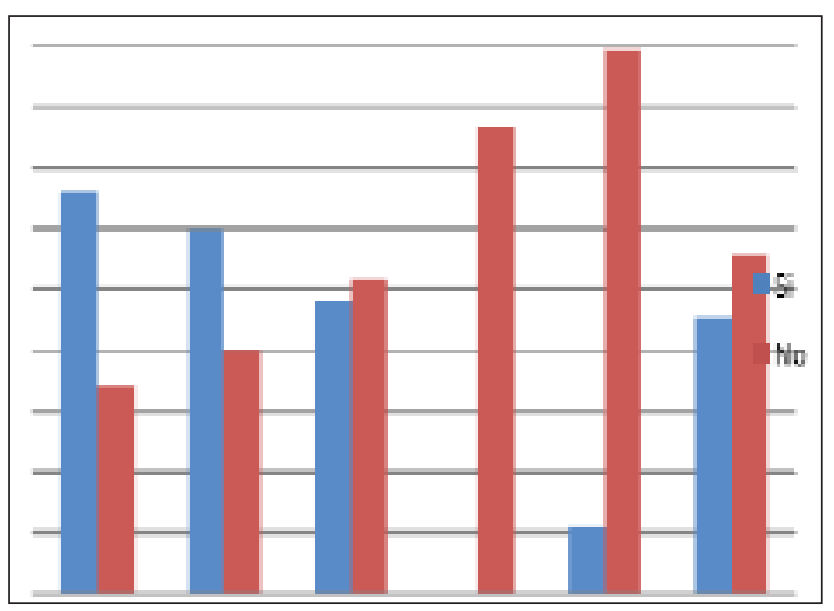

Figura 2. Consumo de Pisco

Fuente: Investigación de mercado Sept. 2014.

\section{Canales}

Canal directo, en el que el productor (de las zonas con D. O.) se encarga de trasladar el producto hasta las manos del consumidor y lo hace mediante la venta directa en bodega y/o en sus locales comerciales de venta de pisco; el otro mecanismo es de la bodega a la feria. En todos los casos el producto sale de manos del productor a las manos del cliente final (Figura 3).

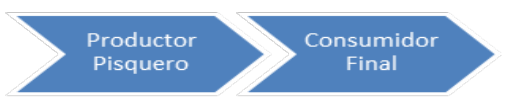

Figura 3. Canal Directo

Canal corto, aquí la comercialización del pisco es desarrollada principalmente por el detallista denominado tienda especializada o licorería, retail (supermercados) y tiendas de grifos, este canal se desarrolla por la fuerte concentración del intermediario para llegar al cliente final, cuentan con cadenas de tiendas y su presencia es la más próxima al consumidor (Figura 4).

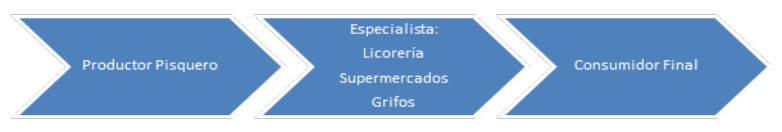

Figura 4. Canal Corto

Canal largo, los productores llegan al consumidor final mediante los servicios que brindan mayoristas y minoristas, denominando al mayorista distribuidor principalmente en las zonas en donde el productor no puede llegar, y logrando la cobertura de esos mercados mediante la participación de minorias del tipo licorerías pequeñas, restaurantes, discotecas y bares, este canal permite un mayor ingreso a puntos de venta finales, logrando satisfacer a una demanda altamente fragmentada (Figura 5). 


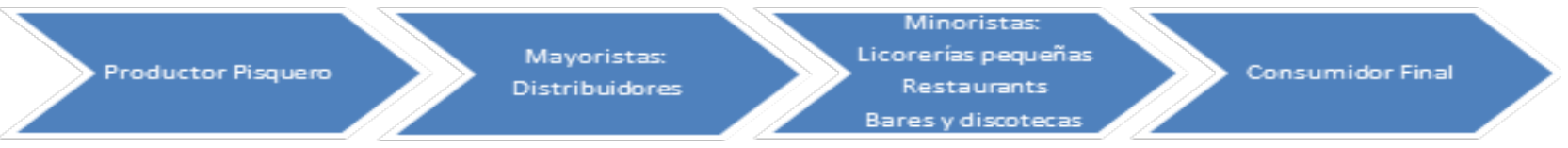

Estos tres tipos de canales conviven en el Perú, en algunas zonas, unos más que otras, su predominancia dependerá del tipo de mercado a tender y de las exigencias del consumidor, sin embargo es importante destacar el rol del retail que ha permitido una vidriera y mayor exposición de las diferentes marcas de piscos a nivel nacional, cuyo crecimiento es sostenido en los últimos años así lo afirma el Departamento de Estudios Económicos del Scotia Bank (2003), la entidad bancaria precisó que el incremento de las ventas en retails se debió a una mayor penetración de las principales cadenas de supermercados -Cencosud (Metro y Wong), Supermercados Peruanos (Plaza Vea, Vivanda, Mass y EconoMax), Macro y Falabella (Tottus)con la apertura de nuevas tiendas en Lima y provincias. En donde se reconoce que las ventas seguirían siendo influenciadas por la capacidad adquisitiva de la población y la disponibilidad de los créditos de consumo. Al término del 2013, el número total de locales en operaciones del sector supermercados fue de 225 , de los cuales 154 están en Lima y 71 en provincias; cuyo crecimiento se proyecta sostenido dado que el mercado aún no está coberturado al $100 \%$.

En el año 2016 este canal fue el más utilizado por las principales marcas en el mercado interno, cuya presencia permite una exposición permanente a diferencia de las ferias en las cuales predomina la temporalidad y cuyo desarrollo es limitado, es decir que el productor busca llegar a un número más grande de potenciales consumidores, y por el lado de la demanda se observa en la Figura 6 que el supermercado concentra las transacciones principalmente de los segmentos B, C, D y E. Sin embargo, en el segmento A el punto de venta de mayor frecuencia es la licorería con un $69,2 \%$.

Destacándose la conveniencia de este punto de venta por aspectos reconocidos tales como la amplia gama de productos a escoger, el tipo de presentación, las degustaciones y promociones que impulsan la compra, se identifican que los puntos de compra de bebidas alcohólicas con mayor preferencia, son los supermercados y las licorerías, siendo estas últimas la de mayor predominancia con un 33,4\% en promedio (Figura 7).

En el canal de comercialización del pisco para el mercado interno intervienen al inicio de la cadena el productor (se distinguen tres categorías en el mercado formal: la gran empresa, el productor pequeño y el productor artesanal), seguido de los intermediarios su número dependerá de la habilidad del productor en atender a su mercado y su capacidad logística, en ausencia de esta el rol del intermediario se hace más fuerte lo que incide en la formación de un canal más largo, finalmente se encuentra al consumidor limeño, cuyas preferencias de consumo se han visto modificadas por su mayor poder adquisitivo y el conocimiento de una oferta más grande, además de la fuerte presencia de impulso y promoción estatal a la bebida bandera del Perú. Siendo la Norma Técnica Peruana NTP (2006) referente que ha permitido la normalización del sector, y la Ley 28681 (2016) con su reglamentación el escenario legal que a partir del 2009 consolidan un escenario competitivo con estructura para dar soporte al crecimiento del sector.

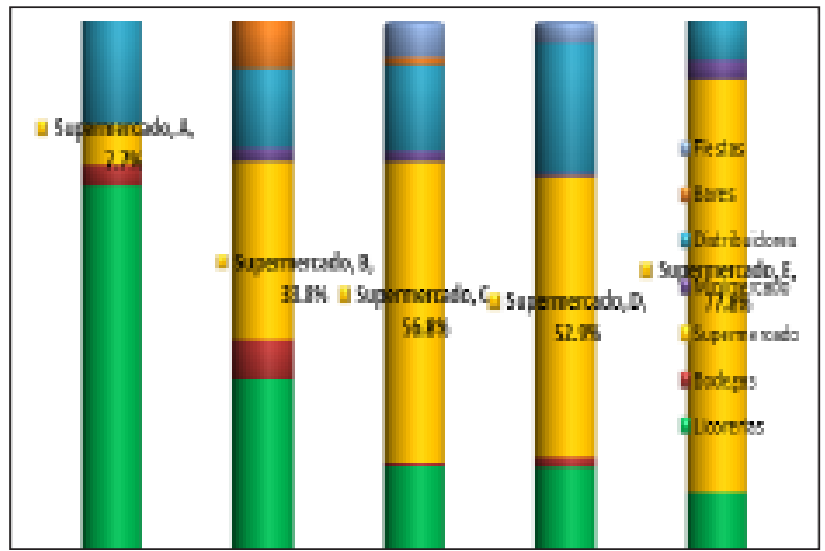

Figura 6. Conveniencia sobre el lugar de compra Fuente: Investigación de mercado Sept. 2014.

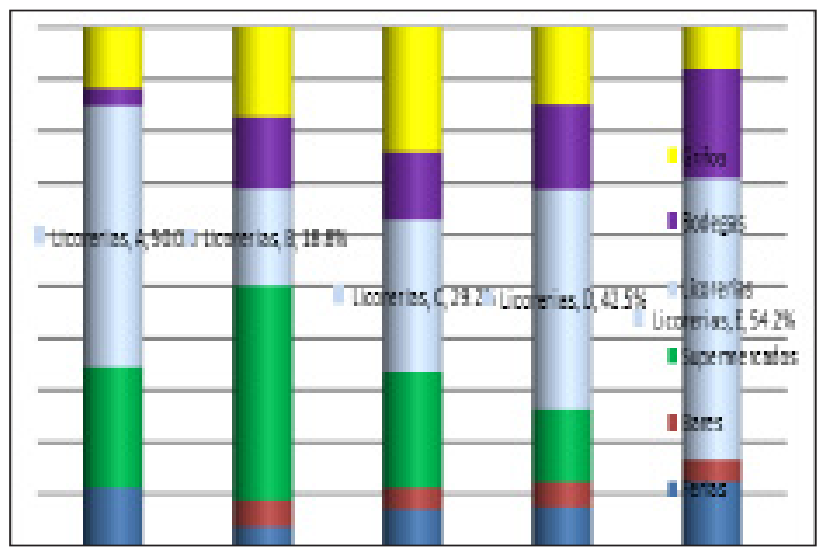

Figura 7. Conveniencia sobre el lugar de compra

Fuente: Investigación de mercado Sept. 2014.

Los factores clave de la comercialización y las estrategias de distribución

Miquel et al. (2012) describen como se va desarrollando la actividad de distribución, partiendo de definir que el comercio realiza la función del intermediario entre los sectores de distribución y consumo. Desde el momento que unos son los que producen y otros los que consumen un bien, necesariamente ha tenido que producirse un intercambio, al menos entre dos partes y además, un acoplamiento del espacio y del tiempo, que separan las actividades de producir dicho bien y de consumirlo. En este intercambio entre las partes, el marketing trata 
de satisfacer necesidades y deseos, e identifica, crea, desarrolla y sirve a la demanda (Lambin, 1998). Mientras la necesidad es una sensación de carencia de algo, el deseo es la forma en la que se expresa la voluntad de satisfacer dicha necesidad. Para poder llevar a cabo este conjunto de actividades, es necesario que existan numerosas personas y entidades que actúan como eslabones intermediarios entre fabricantes y consumidores, formando una trama interdisciplinar de personas y empresas, que constituyen un Sistema Comercial.

El comportamiento de compra del cliente en el punto de venta está influido por una serie de condicionantes internos (necesidades, experiencia y características personales), y externos (factores del entorno) los cuales al ser analizados previamente sirven a la empresa para la elección de la estrategia adecuada, en este sentido las empresas pisqueras deben incorporar aspectos clave como la identificación del target, conocer el mercado y establecer estrategias de distribución.

\section{Identificación del Target}

El mercado meta del pisco son hombres y mujeres mayores de 21 años, de los estratos socioeconómicos A, B y C, residentes en Lima, sociables, dedican tiempo y esfuerzo a actividades lúdicas, comparten entre amigos y disfrutan de nuevas experiencias, son sensoriales (Figura 8). En este segmento se destacan cuatro grupo: Las mujeres, cuya preferencia sobre los piscos es elaborada, es decir buscan preparaciones tipo cocteles y aperitivos, la decoración y presentación es un factor que aprecian, así como la delicadeza del sabor y grado alcohólico. Los Jóvenes entre 21 y 28 prefieren las bebidas de bajo costo en donde no está presente la marca en la decisión de compra. Los Adultos entre 29 y 44 años, su preferencia al momento de compra es racional, buscan nuevos beneficios y la diferenciación por marca, en su mayoría son conocedores de bebidas, buscan estilo y distinción. Y los Adultos de 45 años a más son conocedores de una oferta amplia de productos, exigentes en bondades del producto, empaque y marca, buscan la tradición y rescatan los valores artesanales.

Conocimiento del mercado, gustos y preferencias del consumidor final

La bebida alcohólica de mayor preferencia es la cerveza quien en promedio llega al $47,5 \%$ en todos los segmentos, seguida del wisky con una presencia que supera el $30 \%$ en los segmentos A y B, seguido del pisco en los segmentos A, $\mathrm{B}$ y $\mathrm{C}$ del vodka y finalmente el ron.

El dinamismo del mercado local y las bajas barreras al ingreso han incidido en la comercialización cada vez más de una mayor cantidad de marcas de vinos importados.

En el 2011 se registró el ingreso de cerca de 600 marcas, procedentes de 380 bodegas, en contraste con las 440 importadas en el 2007. Chile fue el principal proveedor de vinos, las marcas argentinas lideraron el mercado en cuanto a importados al concentrar el $31 \%$ del total de marcas, seguidas de las españolas con un $23 \%$, $25 \%$ Chilenas y finalmente un $11 \%$ fueron de procedencia italiana (Prochile, 2012).

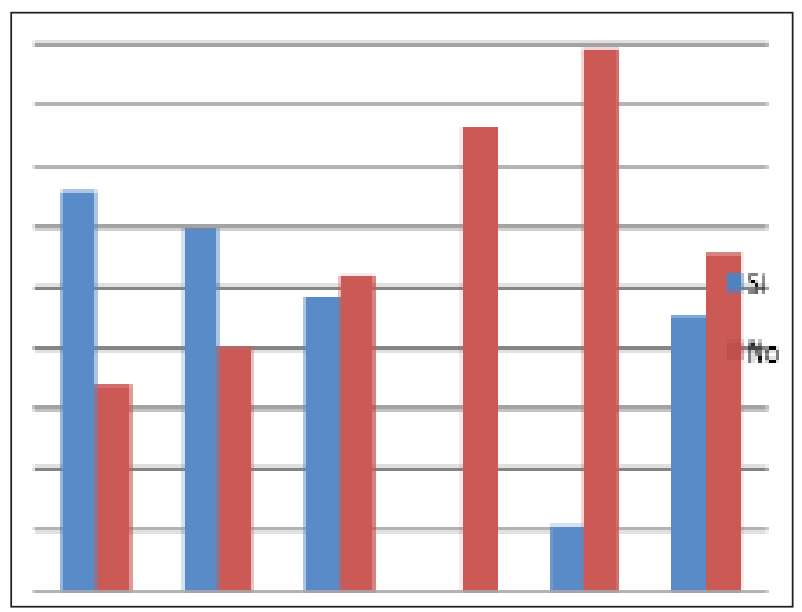

Figura 8. Consumo de pisco

Fuente: Investigación de mercado Sept. 2014.

El consumidor limeño tiene preferencias bien definidas en la forma de realizar sus comprar, se identifica la preferencia por una presentación del producto más elaborada, es decir que la botella no es suficiente, existe una mejor aceptación cuando el producto viene en caja para los niveles A, B y C la preferencia está sujeta a la presentación.

En cuanto a la variedad del pisco a consumir se reconoce que la estrella es la variedad quebranta, por su flexibilidad en las diferentes formas de uso y la recordabilidad de esta sobre las otras variedades. En la Figura 9, de las preferencias se observa que la variedad que le sigue en los estratos A, B y C es el pisco Acholado, y en tercer lugar en el mismo segmento se aprecia a la variedad Italia aunque con mayor presencia en el segmento A.

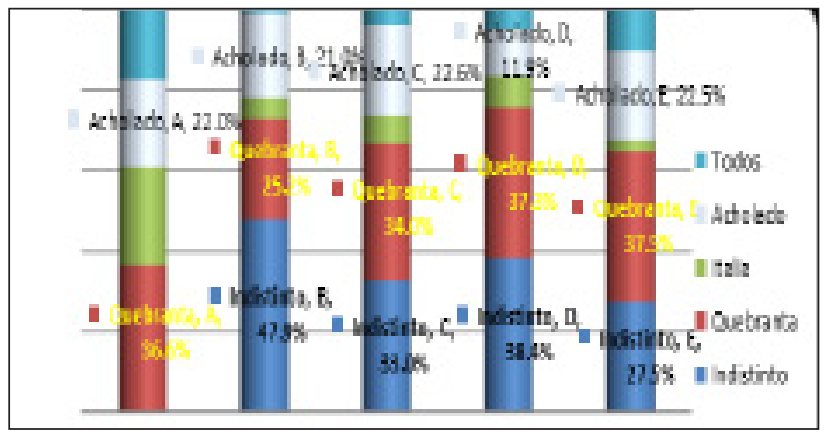

Figura 9. Preferencias sobre la variedad de pisco

Fuente: Investigación de mercado Sept. 2014.

Siendo el atributo buscado en el consumidor limeño sensaciones organolépticas asociadas a un mayor conocimiento y la actividad de cata que se realiza con mayor frecuencia en los segmentos altos, seguido de la comprobación del comentario tradicional "todo buen 
pisco tiene cordón y rosa" y finalmente se vuelve a buscar presentación, en este sentido la empresa no solo debe estar satisfecha con la calidad del producto y el cumplimiento con la normativa existente sino que debe aportar por el esfuerzo de marketing y lograr una presentación que sobresalga en vidriera del punto final de venta. Así lo han demostrado las marcas más exitosas cuyas presentaciones hacen uso de imagen, información y estilo en sus presentaciones. El consumidor de pisco hace la elección de la bebida sobre toda la oferta tanto nacional como internacional en función de tres aspectos principales: la desgustación del producto, el servicio que recibe en el establecimiento y la información que logre obtener del producto, es decir es un tipo de consumidor racional, exigente y cada ves con mayor conocimiento del universo pisquero, propio de los segmentos A, B y C.

\section{Estrategias de Distribución}

Seleccionar el canal de distribución en función de la oferta productiva del productor solo o en agrupación. Establecer planes de acopio para sostener la dotación de oferta de forma permanente en el mercado meta. Mantener el primer año un marketing agresivo en los puntos de venta finales: degustación, información de formas de uso consumo. Desarrollo de la marca en el canal. Gestión de indicadores. Variación del volumen de venta, clasificación de productos por rotación e identificación de clientes. Es en este marco que abordando las condiciones antes descritas la empresa pisquera ya sea independientemente o en forma asociativa podra vencer las barreras existentes en cuanto a la comercialización.

A la fecha el consumidor reconoce algunas marcas, sin embargo no existe aún la fidelización por una en particular, en términos generales los atributos del pisco están asociados a ejercicios de demostración, de experiencia, de nacionalismo, es decir se basan en un enfoque genérico, es aquí donde la empresa debe intervenir buscando desarrollar el mercado de consumo, ya el sector público a través de diferentes programas han logrado un avance interesante principalmente en cuanto a la normatividad y regulación bajo estándares de calidad, quedando pendiente aún el tema del impulso del consumo de pisco.

Las condiciones del entorno han favorecido el ingreso diversas marcas de bebidas sin embargo en el rango de las espirituosas el pisco presenta una oportunidad muy grande como ya lo menciono la investigación de Prochile en el Perú hay oportunidad para el vino y así se demuestra el éxito en los eventos establecidos ya hace algunos años atrás, y en estos el pisco es un invitado de honor.

Se debe mencionar que al año 2013 el consumo de pisco percápita ascendió a $300 \mathrm{ml}$, nivel bajo, para un mercado que va dirigido a la exportación y al consumo interno. El Perú exporta actualmente a 27 países, siendo los principales paises EE.UU. y Chile; mientras que en el mercado interno se han desarrollado, en los últimos siete años, la presencia de marcas líderes, consideradas empresas de gran tamaño.
Se denota una concentración del mercado del pisco, con un pequeño número de empresas, específicamente cuatro las que concentran un $53 \%$ del total de la producción. El pisco compite con sustitutos que son las otras bebidas alcohólicas: whisky, ron, vodka y la cerveza, cuyos mercados se han desarrollado ágilmente, así al 2014, el consumo per cápita anual de cerveza es de aproximadamente 44,5 litros, mientras que el consumo de las demás bebidas alcohólicas es de 1,2 litros ( 0.5 litros de pisco; 0,4 litros de ron; 0,2 litros de whisky y 0,1 litros de vodka), según datos Maximine.

La orientación mantiene una misma dirección, la comprensión del problema identifica acciones claras en una actividad de ingreso al mercado en el libro de "Distribución Comercial" Miquel et al. (2012) describen que los canales de comercialización se debe basar en criterios ajustados al tipo de mercado y las necesidades de la empresa, en donde se considera como un punto inicial la longitud del canal En el actual contexto en donde la tecnología facilita los procesos de comunicación de la empresa con sus mercados a atender, es entonces necesario que la actividad de distribución no solo utilice canales tradicionales en donde el rey en el retail, imponiendo barreras para los pequeños productores: volumen, márgenes de distribución, sobre costos comerciales y financieros. Es un aliado el canal electrónico por sus características, el mismo que viene siendo usado por una de las marcas líderes que ha logrado en tres años consolidar su marca, el pisco Porton quien usa las redes sociales como mecanismo interactivo sumados a diferentes campañas promocionales y apariciones en eventos.

Lambin (1998) "Marketing Estratégico" plantea una línea de acciones en la distribución en donde se entiende que la cobertura se debe gestionar desde la empresa productora, y esta gira en función de su estrategia, para el Pisco Orovilca la presencia en licorerías sumada a medios electrónicos y presencia en eventos hacen a su enfoque de comunicación y exhibición dentro de tres tipos de canales, el directo mencionado anteriormente, en indirecto de un eslabón y el canal electrónico de alta interacción.

\section{Conclusiones}

El sector ha sido liderado por un reducido grupo de marcas líderes, sin embargo estas se centran en la cobertura y la exhibición del producto, existe un entorno favorable dadas las condiciones económicas y culturales, basadas en un mejor nivel de ingresos, la mayor disposición de compra del consumidor final, el incremento de en proporción de los segmentos A B y C.

En el diagnóstico se destacan que los canales de comercialización de Lima Metropolitana se centran en mecanismos tradicionales en donde el supermercado es un participante que domina el ingreso, considerado ideal por diversas marcas ya que una de sus características es la llegada a un número amplio de potenciales compradores, del mismo modo el crecimiento de las licorerías y tiendas especializadas, convirtiéndose en un punto de venta 
potencial y de promoción los lugares de esparcimiento como bares y discotecas.

Las estrategias de distribución exigen que la empresa aprenda y adapte pensando superar barreras y considerando la capacidad de la empresa para establecer su ingreso a través de las los puntos de venta como licorerías, el punto de venta directo y canal alternativo (electrónico) Las estrategias básicas en la distribución se centran en un enfoque que cubra las exigencias del consumidor final con el impulso al producto, y el sostenimiento de la oferta.

\section{Literatura citada}

Achura, O. 2005. Gestión de la cadena de suministros de la bodega de licores Quintal Normal. Tesis de grado, Universidad de Chile, Santiago Chile, Chile. 146pp. Disponible en: http://repositorio.uchile.cl/bitstream/ handle/2250/101802/achurra_m.pdf.

Calderón, M. 2000. Potencialidades de exportaciones de productos alimenticios procesados: tecnología, economía y mercado. Tesis de grado, Universidad Nacional Agraria La Molina, Lima, Perú.

CITE Agroindustrial. 2014. CITE Agroindustrial de Ica ha beneficiado a más de cinco mil productores. Disponible en: http://rpp.pe/economia/economia/ citeagroindustrialde-ica-ha-beneficiado-a-mas-de-5milproductoresnoticia-743130.

Díaz, R. 2006. Análisis del mercadeo interno como estrategia de competitividad en las empresa modernas. Tesis de grado. Universidad de Oriente, Maturín Venezuela. 46pp. Disponible en: http://ri.bib.udo. edu.ve/bitstream/123456789/675/1/TESIS-658.83 D682_01.pdf.

Hernández, B. 1997. Metodología de la Investigación”. 4ta Ed. México; Mc Graw Hill.

Lama, M. 2006. Estrategias de distribución del pisco peruano en el mercado de Estados Unidos”. Tesis de Maestría, Lima, Perú.

Lambin, J. 1998. Marketing Estratégico. 3ra ed. Madrid; Mc Graw Hill.

Ley 28681. Ley que regula la Comercialización, Consumo y Publicidad de Bebidas DECRETO SUPREMO N 012-2009-SA. Disponible en: http:// www.munisullana.gob.pe/new/archivos/licencias funcionamiento/legislacion/.1LEY\%2028681\%20 REGLAMENTO \%2 0LAS \%2 0BEBIDAS \% 20 ALCOH\%C3\%93LICAS\%202009.pdf.

Miquel, P.; Parra, S.; Guerrero, F. y Hermie, C. 2012. Logística y distribución. Sexta ed. España; ESIC.

Monge, R. 2003. Logística de exportación marítima de productos perecibles refrigerados. Trabajo Monográfico, Universidad Nacional Agraria La Molina, Lima, Perú.

Norma Técnica Peruana. 2006. Disponible en: http:// www.elpiscoesdelperu.com/boletines/enero2007/ INDECOPIPISCO.pdf.
Prochile. 2012. Perspectivas de la Industria Alimentaria: El sabor de lo que vendrá. Disponible en: https:// www.prochile.gob.cl/noticia/2012-perspectivas-delaindustria-alimentaria-el-sabor-de-lo-que-vendra/.

Scotiabank. 2003. Reportes de Estudios Económicos. Disponible en: https://www.scotiabank.com.pe/ Acerca-de/Scotiabank-Peru/Mas-informacion/ reportesde-estudios-economicos. 